\title{
El zorro gris pampeano (Lycalopex gymnocercus) como dispersor de las semillas de mora (Morus nigra)
}

\author{
Candela Duarte Baschini ${ }^{1}$ (D) Claudia M. Dellafiore ${ }^{1}$ (D) \\ 1. Departamento de Ciencias Naturales, Universidad Nacional de Río Cuarto - Ruta Nacional 36, km 601, Córdoba, \\ Argentina; cdbaschini@gmail.com; cdelaflor7@hotmail.com
}

Recibido 28-VIII-2020 • Corregido 17-XI-2020 • Aceptado 19-XI-2020

DOI: https://doi.org/10.22458/urj.v13i1.3171

\begin{abstract}
The pampean gray fox (Lycalopex gymnocercus) as disperser of blackberry seeds (Morus nigra)". Introduction: In the mountain forests of the Córdoba, Argentina, foxes (Lycalopex gymnocercus) include in their diet fruits of exotic species such as blackberries (Morus nigra), an invasive species native of Asia. Objective: To learn if blackberry seeds suffer damage in the digestive tract of foxes, and if this affects the viability, germination power, onset and germination rate. Methods: Blackberry seeds were collected in the field and from fresh feces of captive foxes; 300 seeds were randomly selected per treatment and kept in a germinator at $25^{\circ} \mathrm{C}$. The germination criterium was the emergence of the radicle. Results: All the seeds extracted from the feces were healthy. The onset of germination occurred on the same day in both treatments, even though germination rates were higher for the seeds extracted directly from the fruits. Conclusions: Despite minor damage to the seeds, the fox is a disperser of blackberry seeds.
\end{abstract}

Keywords: Lycalopex gymnocercus, gray fox, Morus nigra, blackberry, endozoochory, dispertion.
RESUMEN. Introducción: En los bosques serranos de Córdoba, Argentina, los zorros (Lycalopex gymnocercus) incluyen en su dieta frutos de especies exóticas como la mora (Morus nigra), una especie invasora originaria de Asia. Objetivo: Conocer si las semillas de mora sufren daños en el tracto digestivo de los zorros, y si esto afecta su viabilidad, poder de germinación, inicio y tasa de germinación. Métodos: Recolectamos semillas de zarzamora en el campo y de heces frescas de zorros cautivos, seleccionando al azar 300 semillas por tratamiento que mantuvimos en germinador a $25^{\circ} \mathrm{C}$. El criterio de germinación fue la salida de la radícula. Resultados: Todas las semillas extraídas de las heces estaban sanas. El inicio de la germinación ocurrió el mismo día en ambos tratamientos, aunque las tasas de germinación fueron mayores para las semillas extraídas directamente de los frutos. Conclusiones: A pesar de daños menores a las semillas, el zorro es un dispersor de semillas de mora.

Palabras clave: Lycalopex gymnocercus, zorro, Morus nigra, mora, endozoocoria, dispersión.

El zorro gris pampeano (Lycalopex gymnocercus) es un cánido silvestre nativo de Sudamérica, que en Argentina presenta una gran abundancia y una amplia distribución (Luengos, 2003). Este carnívoro posee una dieta omnívora con un elevado consumo de frutos los cuales pueden llegar a constituir más del 50\% de la misma (Nuñez \& Bozzolo, 2006; Varela, CormenzanaMéndez, Krapovickas \& Bucher, 2008). En los bosques serranos de la provincia de Córdoba se ha observado que los zorros consumen tanto frutos de especies nativas como de especies exóticas entre los que se encuentran los frutos de mora (Morus nigra) (obs. pers.). Esta especie pertenece a la familia Moraceae, es nativa de Asia Menor y se distribuye en regiones tropicales y subtropicales de ambos hemisferios; en Argentina fue introducida con fines ornamentales llegando a convertirse en una planta invasora (Tolaba \& Novara, 1996). 
Morus nigra florece y fructifica en primavera (octubre y noviembre) y cada fruto posee entre 15 a 32 semillas en su interior de 2,5mm de largo por $1 \mathrm{~mm}$ de ancho promedio (obs. per.). Al ser pequeñas, son fáciles de tragar por los animales frugívoros, lo cual puede resultar ventajoso a la hora de dispersarse (Galindo, 1998). Sin embargo, el hecho de que estos frutos sean consumidos por los zorros no implica necesariamente que sus semillas sean dispersadas legítimamente, es decir sanas y/o viables (Fleming \& Sosa, 1994) debido a que el paso a través del tracto digestivo puede afectarlas negativamente, ya sea en forma mecánica y/o afectando su viabilidad.

El presente estudio tuvo por objetivos conocer si las semillas de $M$. nigra sufren daño al pasar a través del tracto digestivo de los zorros y verificar si dicho paso afecta la viabilidad, el poder germinativo, el inicio y tasa de germinación.

\section{MATERIALES Y MÉTODOS}

Para estudiar el efecto del paso de las semillas a través del tracto digestivo del zorro se comparó la germinación entre semillas recolectadas directamente de los frutos y semillas obtenidas de fecas del zorro. Para ello se recolectaron frutos de mora a orillas del Río Chocancharava en la zona noreste de la ciudad de Río Cuarto, provincia de Córdoba, Argentina.

El área de estudio posee una superficie de 12 hectáreas y la vegetación presente corresponde a la región fitogeográfica pampeana - distrito occidental (Cabrera, 1976). La temperatura media anual es de $18^{\circ} \mathrm{C}$ con una precipitación media anual de $800 \mathrm{~mm}$. En total se recolectaron 2000 frutos de $M$. nigra de 10 plantas diferentes.

Se seleccionaron al azar 1000 frutos a los cuales se les extrajeron las semillas. Posteriormente, se seleccionaron al azar 100 semillas a las cuales se les realizó el test de viabilidad de tetrazolio y 300 semillas las cuales fueron puestas a germinar en 10 placas de Petri de 30 semillas cada una. El resto de los frutos (1000) se utilizaron para alimentar a tres zorros en cautiverio del Parque Ecológico Urbano de la ciudad de Río Cuarto (PEU). Al día siguiente se recolectaron las fecas del recinto, y se llevaron al laboratorio donde fueron desarmadas cuidadosamente a mano.

Todas las semillas extraídas fueron analizadas bajo lupa estereoscópica para registrar posibles daños físicos (quiebres y/o exposición del embrión.). Se seleccionaron al azar 300 semillas extraídas de frutos y 300 semillas extraídas de fecas. En cada tratamiento, se sembraron 10 placas de Petri de 30 semillas cada una.

Las placas de Petri de las semillas de frutos y de las fecas del zorro se colocaron en germinador a temperatura constante de $25^{\circ} \mathrm{C}$ y se regaron diariamente. Dichas placas se revisaron cada dos días, durante 100 días, en búsqueda de evidencias de germinación. El criterio de germinación fue la emergencia de la radícula.

El inicio de la germinación (GS) y la tasa de germinación (GR) fueron evaluadas siguiendo el método de Izhaki \& Ne'eman (1997). De acuerdo con estos autores el inicio de la germinación se define como el intervalo (días) entre la siembra y la germinación y se calculó como:

$$
\mathrm{GS}=1 / 6 * \mathrm{P}
$$

donde $\mathrm{P}$ es el porcentaje final de germinación.

La tasa de germinación se calculó como:

$$
G R=(5 / 6 * P) /(T 2-T 1)
$$

donde T1 es el intervalo de tiempo (días) entre la siembra y la germinación de $1 / 6 * \mathrm{P}$ de las semillas, y $\mathrm{T} 2$ es el intervalo de tiempo (días) entre la siembra y la emergencia de $5 / 6 * \mathrm{P}$ de plántulas. 
El poder germinativo (PG) representa el porcentaje final de semillas que germinan $(\mathrm{Sg})$ con relación al número total de semillas sembradas (Ss).

$$
P G=(S g / S s) \times 100 \%
$$

Se calculó el porcentaje acumulado de germinación entre las semillas de los frutos y las fecas a lo largo del tiempo. Para los análisis estadísticos se utilizó la prueba de la t para muestras independientes para detectar diferencias significativas entre los tratamientos.

Por último, se aplicó la prueba de tetrazolio a las semillas que no germinaron al finalizar el experimento para conocer su viabilidad (Cottrell, 1947; MacKay, 1972). Para ello las semillas fueron cortadas por la mitad y se sumergieron en una solución acuosa al 0,1\% de cloruro de 2, 3, 5 - trifenil - tetrazolio, $\mathrm{pH} 7$, en la oscuridad durante 72 horas a una temperatura constante de $25^{\circ} \mathrm{C}$. Finalmente, el embrión se observó bajo lupa estereoscópica; los teñidos de rojo eran viables y los no coloreados eran inviables (Bradbeer, 2013).

\section{RESULTADOS}

El $100 \%$ de las semillas recolectadas de las fecas de zorro estaban sanas; es decir no presentaron evidencias de daño físico luego de pasar por el tracto digestivo de los animales.

El $100 \%$ de las semillas provenientes de los frutos estaban vivas previo a comenzar el experimento.

El inicio de germinación (GS) fue en el día tres tanto para las semillas provenientes de los frutos como para las obtenidas de las fecas de los zorros ( $t=-1,24 ; p=0,23 ;$ Fig. 1).

Los porcentajes de germinación acumulada de las semillas de los frutos y las obtenidas de las fecas de los zorros fueron significativamente diferentes ( $t=16,33 ; p<0,0001 ;$ Fig. 1). Esta diferencia entre los tratamientos se debería a diferencias en los porcentajes de germinación (PG) y a la tasa de germinación (GR). El PG fue significativamente mayor para las semillas de los frutos (PG= $82,5 \pm 3,41)$ que para las semillas de las fecas de zorro ( $P G=52,3 \pm 4,42)(t=5,42 ; p<0,0001 ;$ Fig. 1$)$. La tasa de germinación (GR) fue mayor para las semillas de los frutos $(13,75 \pm 4,27)$ que para las semillas de las fecas $(3,96 \pm 3,85)$, mostrando diferencias significativas entre ellas $(t=2,39 ; p=0,02)$.

Al finalizar el experimento 105 semillas obtenidas de los frutos $(17,5 \%)$ y 286 semillas obtenidas de las fecas $(47,7 \%)$ no habían germinado. A dichas semillas se les aplicó la prueba de tetrazolio para conocer su viabilidad y se encontró que el $47 \pm 9 \%$ y $21 \pm 4 \%$ de las semillas de los frutos y fecas respectivamente estaban vivas. Estos resultados muestran diferencias estadísticamente significativas $(t=-2,68 ; p=0,02)$. 


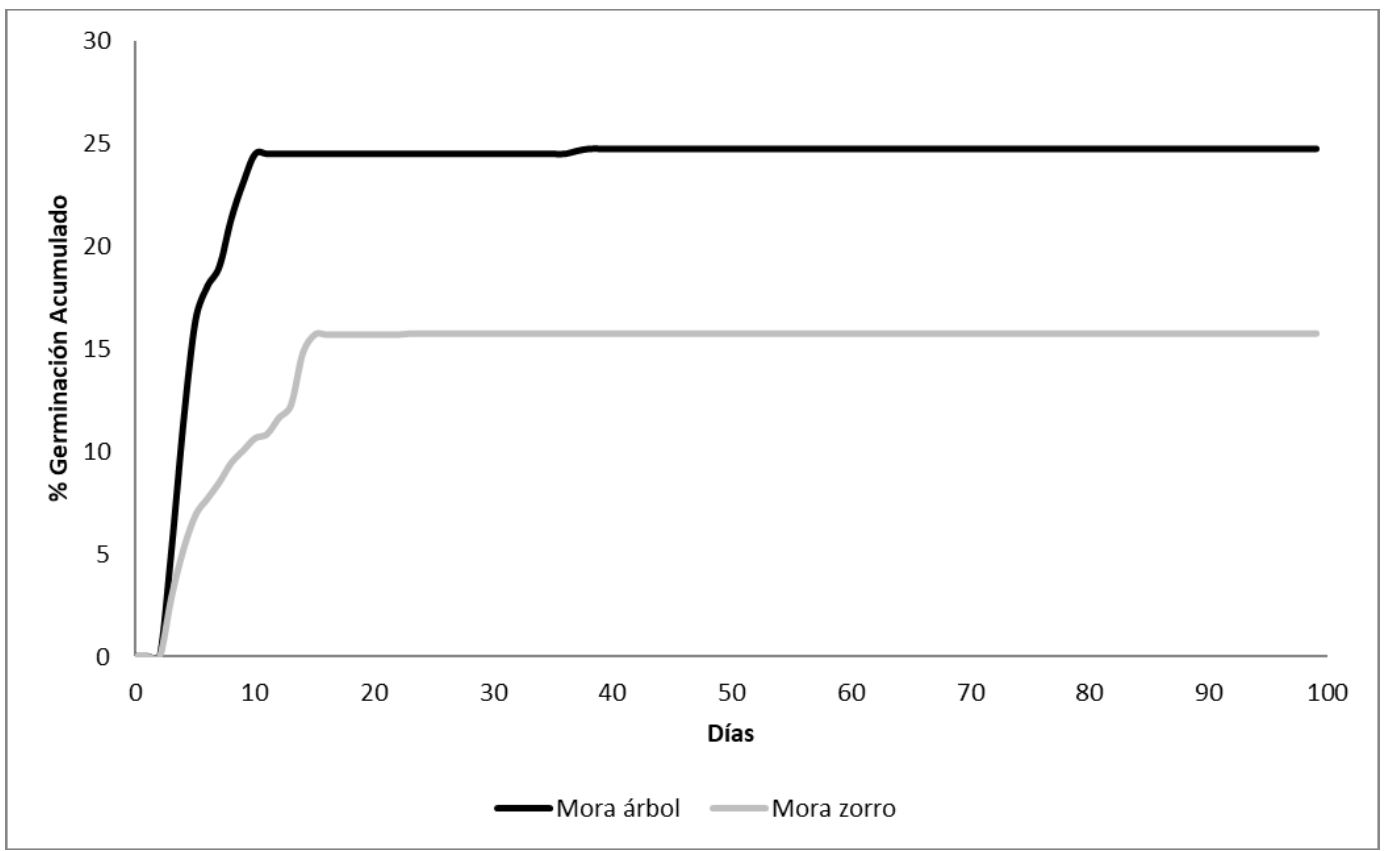

Fig. 1. Porcentaje de germinación acumulado de las semillas de moras extraídas de árboles (línea negra) y de las semillas de moras extraías de las fecas de zorro (línea gris) a lo largo de 100 días.

\section{DISCUSIÓN}

Las semillas de Morus nigra no sufren daño al pasar por el tracto digestivo del zorro, lo cual podría deberse a que las semillas son muy pequeñas en relación al tamaño de la boca de estos carnívoros, lo que permitiría que las semillas ingeridas sean generalmente defecadas sin daños mecánicos (Herrera, 1989; Galindo, 1998; Suárez-Esteban, Delibes, \& Fedriani, 2013). De acuerdo con estos resultados, el zorro sería un dispersor legítimo de $M$. nigra.

El inicio de la germinación fue similar para las semillas de los frutos que para las de las fecas de los zorros, ya que en ambos casos comenzaron a germinar poco tiempo después de ser sembradas. Resultados similares se han observados en otros carnívoros donde el $73 \%$ de las especies de frutos consumidos no manifiestan diferencias en el inicio de germinación (Lieberman \& Lieberman, 1986; Traveset, 1998). El poder germinativo fue significativamente mayor cuando las semillas se extrajeron directamente de los frutos que las provenientes de las fecas. Lo mismo ocurrió con la tasa de germinación que, si bien en ambos casos sigue un patrón de crecimiento exponencial los primeros días y luego se detiene, es mayor para las semillas extraídas de los frutos que para las que fueron consumidas por los zorros.

Esto podría estar indicando que existe algún factor en el tracto digestivo de estos mamíferos que provoca una disminución en el número de semillas germinadas y una reducción en la velocidad a la que lo hacen. Dichos resultados, sumados al hecho de que las semillas de las fecas no presentaron daños externos y que el número de semillas viables al finalizar el estudio fue significativamente menor para las provenientes de las fecas, nos llevan a suponer que en el tracto digestivo de los zorros ocurre algún proceso químico que resulta negativo para las semillas de mora.

Diversos estudios han demostrado que el tamaño de una semilla puede determinar el tiempo que permanece en el tracto digestivo de un animal (Levey \& Grajal, 1991; Izhaki \& Arad, 1995), y que las semillas de menor tamaño tienden a estar más tiempo en el sistema digestivo, por lo cual es más probable que sufran daños por abrasión (Traveset, 1998). Estos resultados coinciden con el $17 \%$ de los estudios sobre el efecto de la endozoocoria, donde el paso por el tracto digestivo 
de diferentes animales perjudica la germinación de las semillas (Traveset, 1998). Mayores estudios son necesarios para conocer cuál o cuáles son las causas específicas de esta disminución en el poder germinativo y tasa de germinación.

M. nigra, como la mayoría de las plantas exóticas, genera una gran cantidad de frutos y cada uno de ellos puede tener hasta 32 semillas (obs. pers.), por ende, el consumo de una pequeña cantidad de frutos por parte del zorro implicaría que un número considerable de ellas estén siendo dispersadas sanas. Esto sumado a la amplia movilidad de este mamífero (Luengos, 2003) haría que las semillas consumidas puedan llegar a sitios alejados de la planta madre, lo cual le conferiría numerosos beneficios, ya que este transporte disminuye la competencia intra-específica (Galindo, 1998) y el riesgo de depredación (Janzen, 1970; Fleming \& Heithaus, 1981; Fleming \& Williams, 1990). La llegada de las semillas a áreas lejanas a la planta madre promovería, además, el flujo genético entre poblaciones de plantas (Heithaus, 1982). Es decir que, a pesar de que el zorro disminuye su poder germinativo y tasa de germinación, la interacción mutualista sería positiva para la planta ya que el zorro realizaría una importante lluvia de semillas y permitiría el ingreso de esta especie a nuevas áreas abiertas a la colonización. Teniendo en cuenta que la mora es una especie exótica invasora, serían necesarios estudios más profundos para conocer el efecto de su dispersión por endozoocoria sobre las especies nativas de la zona, las cuales probablemente se verán perjudicadas.

\section{AGRADECIMIENTOS}

Los autores agradecen a la Secretaría de Ciencia y Técnica de la Universidad Nacional de Río Cuarto por al apoyo financiero.

\section{ÉTICA, CONFLICTO DE INTERESES Y DECLARACIÓN DE FINANCIAMIENTO}

Los autores declaran haber cumplido con todos los requisitos éticos y legales pertinentes, tanto durante el estudio como en el manuscrito; que no hay conflictos de interés de ningún tipo, y que todas las fuentes financieras se detallan plena y claramente en la sección de agradecimientos. Asimismo, están de acuerdo con la versión editada final del documento. El respectivo documento legal firmado se encuentra en los archivos de la revista.

La declaración de la contribución de cada autor al manuscrito es la siguiente: C.D.B., \& C.M.D: recolección de datos, análisis, confección y redacción del manuscrito.

\section{REFERENCIAS}

Bradbeer, J. W. (2013). Seed dormancy and germination. USA: Springer Science \& Business Media.

Cabrera, A. L. (1976). Enciclopedia Argentina de agricultura y jardinería: regiones fitogeográficas argentinas. Argentina: Editorial Acme.

Cottrell, H. J. (1947). Tetrazolium salt as a seed germination indicator. Nature, 159(4048), 748.

Fleming, T. H., \& Heithaus, E. R. (1981). Frugivorous Bats, Seed Shadows, and the Structure of Tropical Forests. Biotropica, 13(2), 45-53.

Fleming, T. H., \& Williams, C. F. (1990). Phenology, seed dispersal, and recruitment in Cecropia peltata (Moraceae) in Costa Rican tropical dry forest. Journal of tropical ecology, 6(2), 163-178. 
Fleming, T. H., \& Sosa, V. J. (1994). Effects of nectarivorous and frugivorous mammals on reproductive success of plants. Journal of Mammalogy, 75(4), 845-851.

Galindo, J. (1998). Dispersión de semillas por murciélagos: su importancia en la conservación y regeneración del bosque tropical. Acta zoológica Mexicana (N.S.), (73), 57-74.

Heithaus E. R. (1982) Coevolution between Bats and Plants. En T.H. Kunz (Ed). Ecology of Bats (pp. 327-367). Springer, Boston, MA.

Herrera, C. M. (1989). Frugivory and seed dispersal by carnivorous mammals, and associated fruit characteristics, in undisturbed Meditarranean. Oikos, 55(2), 250-262.

Izhaki, C., \& Arad, K. Z. (1995). The effect of bat (Rousettus aegyptiacus) dispersal on seed germination in eastern Mediterranean hábitats. Oecologia, 101(3), 335-342.

Izhaki, I., \& Ne'eman, G. (1997). Hares (Lepus spp.) as seed dispersers of Retama raetam (Fabaceae) in sandy landscape. Journal of arid environment, 37(2), 343-354.

Janzen, D. H. (1970). Herbivores and the number of tree species in tropical forests. The American Naturalist, 104(940), 501-528.

Levey, D. J., \& Grajal A. (1991). Evolutionary Implications of Fruit-Processing Limitations in Cedar Waxwings. The American Naturalist, 138(1), 171-189.

Lieberman, M., \& Lieberman, D. (1986). An experimental study of seed ingestion and germination in a plant-animal assemblage in Ghana. Journal of Tropical Ecology, 2(2), 113-126.

Luengos, E. (2003). Estudio comparado de metodologías de captura y estimación de las poblaciones de zorro pampeano Pseudalopex gymnocercus (tesis inédita de maestría). Universidad Nacional del Sur, Bahía Blanca, Argentina.

MacKay D. B. (1972) The Measurement of Viability. En E.H. Roberts (Ed). Viability of Seeds (pp. 172-208). Springer, Dordrecht.

Nuñez, M. B., \& Bozzolo, L. (2006). Descripción de la dieta del zorro gris, Pseudalopex griseus (Canidae) (Gray, 1842), en el Parque Nacional Sierra de las Quijadas, Argentina. Gayana, 70(2), 163-167.

Suárez-Esteban, A., Delibes, M., \& Fedriani, J. M. (2013). Barriers or corridors? The overlooked role of unpaved roads in endozoochorous seed dispersal. Journal of Applied Ecology, 50(3), 767-774.

Tolaba, J. A., \& Novara, L. (1996). Moraceae. Aportes Botanicos de Salta-Serie Flora, 4(9), 1-13.

Traveset, A. (1998). Effect of seed passage throught vertebrate frugivores' guts on germination: a review. Perspectives in a Plant Ecology, Evolution and Systematics, 1(2), 151-190.

Varela, O., Cormenzana-Méndez, A., Krapovickas, L., \& Bucher, E. H. (2008). Seasonal diet of the pampas fox (Lycalopex gymnocercus) in the Chaco dry Woodland, northwestern Argentina. Journal of Mammalogy, 89(4), 1012-1019. 